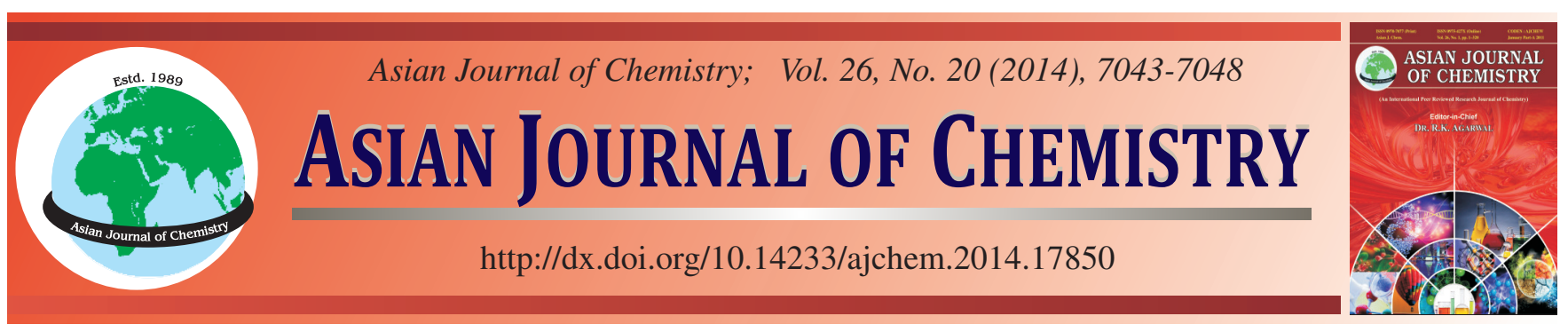

\title{
Effect of Binary Organic Solvent Mixtures on Lipase-Catalyzed Regioselective Acylation of 6-Azauridine with Various Structural Vinyl Esters
}

\author{
Yanhong Bi ${ }^{1}$, Wenying Du ${ }^{1}$, Zhaoyu W Ang $^{1,2,3, *}$, Linghong NiE ${ }^{1}$ and XiRONG ZhaO ${ }^{1}$
}

${ }^{1}$ School of Life Science and Chemical Engineering, Huaiyin Institute of Technology, Huai'an 223003, P.R. China

${ }^{2}$ Jiangsu Provincial Engineering Laboratory for Biomass Conversion and Process Integration, Huai'an 223005, P.R. China

${ }_{3}^{3}$ Jiangsu Provincial Engineering Laboratory for Advanced Materials of Salt Chemical Industry, Huai'an 223003, P.R. China

*Corresponding author: Tel/Fax: +86 517 83591165; E-mail: biowzy@126.com

Received: 18 April 2014;

Accepted: 26 June 2014;

Published online: 25 September 2014;

AJC-16063

In this paper, the regioselective acylation of 6-azauridine for preparing its potential prodrugs was successfully conducted in the hydrophobicity-based binary solvent system using Candida antarctica lipase B. Comparative study showed that the reaction rate, thermal and operational stability of the enzyme were dramatically improved by using the binary mixtures of acetone and isooctane $(85: 15$, v/v) as the reaction medium. In addition, the acyl donor sturcture displayed great influence on the behaviour of the enzyme in binary solvent system, presumably due to their different interactions with the active site of the lipase.

Keywords: Candida antarctica lipase B, 6-Azauridine, Regioselective acylation, Binary solvent system.

\section{INTRODUCTION}

6-Azauridine (AzUrd), a pyrimidine nucleoside analogue, is a well-known antitumour and antiviral agent and has been clinically applied in the treatment of psoriasis and herpes viral infections for many years ${ }^{1,2}$. Investigations on the pharmacokinetics have revealed that 6 -azauridine could inhibit the activity of orotidylate decarboxylase which is a key enzyme in de novo synthesis of pyrimidine ${ }^{3}$. However, like other nucleoside drugs, the polyhydroxy structure of 6-azauridine exhibits various side effects including poor oral bioavailability, difficulty to traverse biological membranes and skin layers and its more toxic metabolite of 6-azauracil. To reduce the toxicity of nucleoside drugs while retaining their pharmacological activity, some strategies for selective modification of nucleosides have been explored to synthesis the potential prodrugs with improved pharmacokinetic profile and therapeutic efficacy ${ }^{4,5}$.

Clinical studies indicated that the fatty acid ester derivatives of nucleoside drugs with the desired lipophilicity were effectively circumvent the drawbacks mentioned above and intended to facilitate the accumulation and increase retention of the drugs in the body ${ }^{6,7}$. However, producing specific position ester derivatives of unprotected polyhydroxy compounds continues to be a challenge to organic chemists via conventional chemical approaches, owing to the existence of several hydroxyl groups with similar chemical reactivity in the molecule ${ }^{8}$. In recent years, enzymatic acylation of nucleosides associated with high regioselectivity, mild reaction conditions and environmental friendliness in nonaqueous media has proved to be a practicable option for this purpose. For example, various kinds of 5'- or 3'-O-acyl-nucleosides derivatives were successfully enzymatic synthesized by our group ${ }^{9-11}$. Furthermore, among the biocatalysts used extensively, $C$. antarctica lipase B have undoubtedly proved to be effective catalysts for a great variety of chemical processes, especially towards acylating the 5'-OH of the sugar moiety of many nucleosides 9 .

However, a general problem usually encountered with the use of Candida antarctica lipase B in acylation of nucleosides is the maintenance of its catalytic activity in strong polar organic solvents, such as the DMF ( $N, N$-dimethylformamide) and DMSO (dimethyl sulfoxide). It is well-documented that although polar solvents are always suited media for hydrophilic nucleosides, the essential water shell of the enzyme would be destroyed, thus disrupting the functional structure of enzyme ${ }^{12}$. Recently, binary organic solvent mixtures of hydrophilic and hydrophobic solvents have proved to be promising as reaction media for maintaining the higher enzymatic activity and stability and advantageous to selectively synthesize the desired ester derivatives using enzymes ${ }^{13,14}$. For these reasons, here we chose the regioselective undecylenoylation of 6-azauridine as a model reaction to examine the influence of the binary organic solvent mixtures on the behaviour of the C. antarctica lipase B. Besides, the general applicability of the binary solvent 
strategy in this acylation was also investigated by using different acyl donors.

\section{EXPERIMENTAL}

Candida antarctica lipase B (an immobilized lipase B from Candida antarctica, $2875 \mathrm{U} / \mathrm{g}$ ) was purchased from Novozymes, Denmark. Vinyl acetate, vinyl chloroacetate, vinyl benzoate and vinyl crotonate were obtained from Alfa Aesar. Vinyl butyrate, vinyl hexanoate, vinyl caprylate, vinyl decanoate, vinyl myristate, vinyl palmitate and vinyl 10-undecenoate were from TCI. 6-Azauridine and vinyl laurate were purchased from Aldrich. All other chemicals were also from commercial sources and of the highest purity available.

Enzymatic acylation of nucleoside: In a typical experiment, 6-azauridine $(0.04 \mathrm{mmol}), C$. antarctica lipase B and vinyl ester were added into $2 \mathrm{~mL}$ anhydrous organic solvent and the mixture was incubated at a predetermined temperature in an orbital air-bath shaker (200 rpm). Aliquots were withdrawn at specified time intervals from the reaction mixture and then diluted 50-fold with corresponding mobile phase prior to HPLC analysis. Regioselectivity was defined as the molar ratio of the desired product to the total amount of ester products formed. All data are averages of experiments performed in triplicate.

Control of the initial water activity: The organic solvents were dried with $4 \AA$ molecular sieves. The enzyme, substrates and organic solvents were put in separate closed containers with different saturated salt solutions for $72 \mathrm{~h}$ to fix the initial water activity $\left(a_{w}\right)$. The following salts were used: $\operatorname{LiBr}\left(a_{w}=\right.$ $0.07), \operatorname{LiCl}\left(a_{w}=0.11\right), \operatorname{MgCl}_{2}\left(a_{w}=0.33\right), \operatorname{Mg}\left(\mathrm{NO}_{3}\right)_{2}\left(a_{w}=\right.$ $0.53), \mathrm{NaCl}\left(\mathrm{a}_{\mathrm{w}}=0.75\right)$ and $\mathrm{KCl}\left(\mathrm{a}_{\mathrm{w}}=0.85\right)^{15}$.

HPLC analysis: The reaction mixture was analyzed by RP-HPLC on a $4.6 \times 250 \mathrm{~mm}(5 \mu \mathrm{m})$ Zorbax SB-C18 column (Agilent Technologies Industries Co., Ltd., USA) using an Agilent G1311A pump and a UV Detector at $263 \mathrm{~nm}$. The mobile phase is a mixture of water and methanol at a flow rate of $1.0 \mathrm{~mL} / \mathrm{min}$. The volumetric ratio of water to methanol and the retention time for 6 -azauridine and its 5 '- $O$-monoester were 90/10, 2.410 and 10.032 min (acetylation), 77/23, 2.363 and $12.160 \mathrm{~min}$ (butyrylation), 30/70, 2.277 and $11.910 \mathrm{~min}$ (decanoylation), 25/75, 2.183 and $13.287 \mathrm{~min}$ (lauroylation), 20/80, 2.229 and 16.169 min (myristoylation), 15/85, 2.208 and $15.979 \mathrm{~min}$ (palmitoylation), 85/15, 2.093 and $8.765 \mathrm{~min}$ (chloroacetylation), 60/40, 2.361 and 8.624 min (pivaloylation), 30/70, 2.161 and $9.900 \mathrm{~min}$ (undecenoylation), 68/ $32,2.398$ and 21.451min (benzoylation) and 70/30, 2.483 and 8.261 min (crotonylation), respectively. For hexanoylation and caproylation, the mobile phase was a mixture of water and methanol at a flow rate of $0.9 \mathrm{~mL} / \mathrm{min}$ and the volumetric ratio (water/methanol) and retention time for 6-azauridine and its $5^{\prime}-O$-monoester were $60 / 40,2.112$ and $12.078 \mathrm{~min}$ (hexanoylation), 38/62, 2.413 and 8.843 min (caproylation), respectively.

Purification and structure determination of the esters derivatives: The enzymatic reactions were scaled up and the reaction mixtures were separated and purified by flash column chromatography using ethyl acetate (EA)/petroleum ether (PE) as the mobile phase. The structure of the ester derivatives was determined by ${ }^{13} \mathrm{C}$ NMR and ${ }^{1} \mathrm{H}$ NMR (Bruker DRX-400 NMR Spectrometer, Bruker Co., Germany) at $100 \mathrm{MHz}$ and 400 $\mathrm{MHz}$, respectively. DMSO- $d_{6}$ was used as the solvent.

6-Azauridine acetate: ${ }^{1} \mathrm{H}$ NMR: $\delta 12.22\left(1 \mathrm{H}\right.$, br s, $\left.\mathrm{H}_{3}\right)$, $7.56\left(1 \mathrm{H}, \mathrm{s}, \mathrm{H}_{5}\right), 5.91\left(1 \mathrm{H}, \mathrm{d}, J=4 \mathrm{~Hz}, \mathrm{H}_{1^{\prime}}\right), 5.37(1 \mathrm{H}, \mathrm{br}$ s, $\left.\mathrm{OH}_{2^{\prime}}\right), 5.19\left(1 \mathrm{H}\right.$, br s, $\left.\mathrm{OH}_{3^{\prime}}\right), 4.27-4.25\left(1 \mathrm{H}, \mathrm{m}, \mathrm{H}_{5^{\prime}-1}\right), 4.19$ $\left(1 \mathrm{H}, \mathrm{m}, \mathrm{H}_{2^{\prime}}\right), 4.05-4.04\left(1 \mathrm{H}, \mathrm{m}, \mathrm{H}_{3^{\prime}}\right), 3.98-3.90\left(2 \mathrm{H}, \mathrm{m}, \mathrm{H}_{4^{\prime}}+\right.$ $\left.\mathrm{H}_{5^{\prime}-2}\right), 1.99\left(3 \mathrm{H}, \mathrm{s}, \mathrm{H}_{2^{\prime \prime}}\right) .{ }^{13} \mathrm{C}$ NMR: $\delta 170.626\left(\mathrm{C}_{1^{\prime \prime}}\right), 156.889$ $\left(\mathrm{C}_{4}\right), 148.619\left(\mathrm{C}_{2}\right), 136.898\left(\mathrm{C}_{5}\right), 90.185\left(\mathrm{C}_{1^{\prime}}\right), 81.359\left(\mathrm{C}_{4^{\prime}}\right)$, $72.954\left(\mathrm{C}_{2^{\prime}}\right), 71.083\left(\mathrm{C}_{3^{\prime}}\right), 64.643\left(\mathrm{C}_{5^{\prime}}\right), 21.029\left(\mathrm{C}_{2^{\prime \prime}}\right)$.

6-Azauridine butyrate: ${ }^{1} \mathrm{H}$ NMR: $\delta 12.21\left(1 \mathrm{H}\right.$, br s, $\left.\mathrm{H}_{3}\right)$, $7.51\left(1 \mathrm{H}, \mathrm{s}, \mathrm{H}_{5}\right), 5.90\left(1 \mathrm{H}, \mathrm{d}, J=4 \mathrm{~Hz}, \mathrm{H}_{1^{\prime}}\right), 5.35(1 \mathrm{H}, \mathrm{br}$ s, $\left.\mathrm{OH}_{2^{\prime}}\right), 5.17\left(1 \mathrm{H}\right.$, br s, $\left.\mathrm{OH}_{3^{\prime}}\right), 4.28-4.24\left(1 \mathrm{H}, \mathrm{m}, \mathrm{H}_{5^{\prime}-1}\right), 4.21-$ $4.20\left(1 \mathrm{H}, \mathrm{m}, \mathrm{H}_{2^{\prime}}\right), 4.05-4.04\left(1 \mathrm{H}, \mathrm{m}, \mathrm{H}_{3^{\prime}}\right), 3.97-3.92(2 \mathrm{H}, \mathrm{m}$, $\left.\mathrm{H}_{4^{\prime}}+\mathrm{H}_{5^{\prime}-2}\right), 2.23\left(2 \mathrm{H}, \mathrm{t}, J=8 \mathrm{~Hz}, \mathrm{H}_{2^{\prime \prime}}\right), 1.52-1.47$ ( $\left.2 \mathrm{H}, \mathrm{m}, \mathrm{H}_{3^{\prime \prime}}\right)$, $0.83\left(3 \mathrm{H}, \mathrm{t}, J=8 \mathrm{~Hz}, \mathrm{H}_{4^{\prime}}\right) .{ }^{13} \mathrm{C} \mathrm{NMR}: \delta 173.079\left(\mathrm{C}_{1^{\prime \prime}}\right), 156.874$ $\left(\mathrm{C}_{4}\right), 148.578\left(\mathrm{C}_{2}\right), 136.782\left(\mathrm{C}_{5}\right), 90.201\left(\mathrm{C}_{1^{\prime}}\right), 81.331\left(\mathrm{C}_{4^{\prime}}\right)$, $72.927\left(\mathrm{C}_{2^{\prime}}\right), 70.970\left(\mathrm{C}_{3^{\prime}}\right), 64.303\left(\mathrm{C}_{5^{\prime}}\right), 35.651\left(\mathrm{C}_{2^{\prime}}\right), 18.279$ $\left(\mathrm{C}_{3^{\prime \prime}}\right) 13.773\left(\mathrm{C}_{4^{\prime \prime}}\right)$.

6-Azauridine hexanoate: ${ }^{1} \mathrm{H}$ NMR: $\delta 12.22(1 \mathrm{H}$, br s, $\left.\mathrm{H}_{3}\right), 7.50\left(1 \mathrm{H}, \mathrm{s}, \mathrm{H}_{5}\right), 5.88\left(1 \mathrm{H}, \mathrm{m}, \mathrm{H}_{1^{\prime}}\right), 5.36\left(1 \mathrm{H}, \mathrm{br} \mathrm{s}, \mathrm{OH}_{2^{\prime}}\right)$, $5.17\left(1 \mathrm{H}\right.$, br s, $\left.\mathrm{OH}_{3^{\prime}}\right), 4.26-4.23\left(1 \mathrm{H}, \mathrm{m}, \mathrm{H}_{5^{\prime}-1}\right), 4.18(1 \mathrm{H}, \mathrm{m}$, $\left.\mathrm{H}_{2^{\prime}}\right), 4.03\left(1 \mathrm{H}, \mathrm{m}, \mathrm{H}_{3^{\prime}}\right), 3.94-3.91\left(2 \mathrm{H}, \mathrm{m}, \mathrm{H}_{4^{\prime}}+\mathrm{H}_{5^{\prime}-2}\right), 2.23$ $\left(2 \mathrm{H}, \mathrm{t}, J=7.2 \mathrm{~Hz}, \mathrm{H}_{2^{\prime \prime}}\right), 1.46\left(2 \mathrm{H}, \mathrm{m}, \mathrm{H}_{3^{\prime \prime}}\right), 1.23-1.17(4 \mathrm{H}, \mathrm{m}$, $\left.\mathrm{H}_{4^{\prime \prime}}+\mathrm{H}_{5^{\prime \prime}}\right), 0.80\left(3 \mathrm{H}, \mathrm{t}, J=6.8 \mathrm{~Hz}, \mathrm{H}_{6^{\prime \prime}}\right) .{ }^{13} \mathrm{C} \mathrm{NMR}: \delta 173.223$ $\left(\mathrm{C}_{1^{\prime \prime}}\right), 156.875\left(\mathrm{C}_{4}\right), 148.582\left(\mathrm{C}_{2}\right), 136.794\left(\mathrm{C}_{5}\right), 90.217\left(\mathrm{C}_{1^{\prime}}\right)$, $81.314\left(\mathrm{C}_{4^{\prime}}\right), 72.946\left(\mathrm{C}_{2^{\prime}}\right), 70.969\left(\mathrm{C}_{3^{\prime}}\right), 64.351\left(\mathrm{C}_{5^{\prime}}\right), 33.758$ $\left(\mathrm{C}_{2^{\prime \prime}}\right), 31.045\left(\mathrm{C}_{4^{\prime \prime}}\right), 24.487\left(\mathrm{C}_{3^{\prime \prime}}\right), 22.187\left(\mathrm{C}_{5^{\prime \prime}}\right), 14.175\left(\mathrm{C}_{6^{\prime \prime}}\right)$.

6-Azauridine carprylate: ${ }^{1} \mathrm{H}$ NMR: $\delta 12.22(1 \mathrm{H}$, br s, $\left.\mathrm{H}_{3}\right), 7.50\left(1 \mathrm{H}, \mathrm{s}, \mathrm{H}_{5}\right), 5.87\left(1 \mathrm{H}, \mathrm{m}, \mathrm{H}_{1^{\prime}}\right), 5.35\left(1 \mathrm{H}, \mathrm{br} \mathrm{s}, \mathrm{OH}_{2}\right)$, $5.17\left(1 \mathrm{H}\right.$, br s, $\left.\mathrm{OH}_{3^{\prime}}\right), 4.24-4.22\left(1 \mathrm{H}, \mathrm{m}, \mathrm{H}_{5^{\prime}-1}\right), 4.16(1 \mathrm{H}, \mathrm{m}$, $\left.\mathrm{H}_{2^{\prime}}\right)$, $4.01\left(1 \mathrm{H}, \mathrm{m}, \mathrm{H}_{3^{\prime}}\right), 3.92-3.90\left(2 \mathrm{H}, \mathrm{m}, \mathrm{H}_{4^{\prime}}+\mathrm{H}_{5^{\prime}-2}\right), 2.22$ $\left(2 \mathrm{H}, \mathrm{t}, J=7 \mathrm{~Hz}, \mathrm{H}_{2^{\prime \prime}}\right), 1.45\left(2 \mathrm{H}, \mathrm{m}, \mathrm{H}_{3^{\prime \prime}}\right), 1.18\left(8 \mathrm{H}, \mathrm{m}, \mathrm{H}_{4^{\prime \prime}}+\mathrm{H}_{5^{\prime \prime}}\right.$ $\left.+\mathrm{H}_{6^{\prime \prime}}+\mathrm{H}_{7^{\prime \prime}}\right), 0.78\left(3 \mathrm{H}, \mathrm{t}, J=8 \mathrm{~Hz}, \mathrm{H}_{8^{\prime \prime}}\right) .{ }^{13} \mathrm{C} \mathrm{NMR}: \delta 173.192$ $\left(\mathrm{C}_{1^{\prime \prime}}\right), 156.875\left(\mathrm{C}_{4}\right), 148.576\left(\mathrm{C}_{2}\right), 136.810\left(\mathrm{C}_{5}\right), 90.194\left(\mathrm{C}_{1^{\prime}}\right)$, $81.277\left(\mathrm{C}_{4^{\prime}}\right), 72.983\left(\mathrm{C}_{2^{\prime}}\right), 70.994\left(\mathrm{C}_{3^{\prime}}\right), 64.450\left(\mathrm{C}_{5^{\prime}}\right), 33.797$ $\left(\mathrm{C}_{2^{\prime \prime}}\right), 31.560\left(\mathrm{C}_{6^{\prime \prime}}\right), 27.055\left(\mathrm{C}_{5^{\prime \prime}}\right), 24.828\left(\mathrm{C}_{4^{\prime \prime}}\right), 22.552\left(\mathrm{C}_{3^{\prime \prime}}\right)$, $22.475\left(\mathrm{C}_{7^{\prime \prime}}\right), 14.333\left(\mathrm{C}_{8^{\prime \prime}}\right)$.

6-Azauridine decanoate: ${ }^{1} \mathrm{H}$ NMR: $\delta 12.25(1 \mathrm{H}$, br s, $\left.\mathrm{H}_{3}\right), 7.51\left(1 \mathrm{H}, \mathrm{s}, \mathrm{H}_{5}\right), 5.90\left(1 \mathrm{H}, \mathrm{d}, J=4 \mathrm{~Hz}, \mathrm{H}_{1}\right), 5.39(1 \mathrm{H}, \mathrm{br}$ $\left.\mathrm{s}, \mathrm{OH}_{2^{\prime}}\right), 5.21\left(1 \mathrm{H}\right.$, br s, $\left.\mathrm{OH}_{3^{\prime}}\right), 4.27-4.24\left(1 \mathrm{H}, \mathrm{m}, \mathrm{H}_{5^{\prime}-1}\right), 4.19$ $\left(1 \mathrm{H}, \mathrm{m}, \mathrm{H}_{2^{\prime}}\right), 4.04\left(1 \mathrm{H}, \mathrm{m}, \mathrm{H}_{3^{\prime}}\right), 3.95-3.92\left(2 \mathrm{H}, \mathrm{m}, \mathrm{H}_{4^{\prime}}+\mathrm{H}_{5^{\prime}-2}\right)$, $2.24\left(2 \mathrm{H}, \mathrm{t}, J=8 \mathrm{~Hz}, \mathrm{H}_{2^{\prime \prime}}\right), 1.49-1.45\left(2 \mathrm{H}, \mathrm{m}, \mathrm{H}_{3^{\prime \prime}}\right), 1.20(12 \mathrm{H}$, m, $\left.\mathrm{H}_{4^{\prime \prime}}+\mathrm{H}_{5^{\prime \prime}}+\mathrm{H}_{6^{\prime \prime}}+\mathrm{H}_{7^{\prime \prime}}+\mathrm{H}_{8^{\prime \prime}}+\mathrm{H}_{9^{\prime \prime}}\right), 0.82(3 \mathrm{H}, \mathrm{t}, J=6.8 \mathrm{~Hz}$, $\left.\mathrm{H}_{10^{\prime \prime}}\right) .{ }^{13} \mathrm{C}$ NMR: $\delta 173.192\left(\mathrm{C}_{1^{\prime \prime}}\right), 156.858\left(\mathrm{C}_{4}\right), 148.561\left(\mathrm{C}_{2}\right)$, $136.764\left(\mathrm{C}_{5}\right), 90.229\left(\mathrm{C}_{1^{\prime}}\right), 81.317\left(\mathrm{C}_{4^{\prime}}\right), 72.955\left(\mathrm{C}_{2^{\prime}}\right), 70.983$ $\left(\mathrm{C}_{3^{\prime}}\right), 64.426\left(\mathrm{C}_{5^{\prime}}\right), 33.797\left(\mathrm{C}_{2^{\prime \prime}}\right), 31.704\left(\mathrm{C}_{8^{\prime \prime}}\right), 29.442-28.878$ $\left(\mathrm{C}_{4^{\prime \prime}}+\mathrm{C}_{5^{\prime \prime}}+\mathrm{C}_{6^{\prime \prime}}+\mathrm{C}_{7^{\prime \prime}}\right), 24.809\left(\mathrm{C}_{3^{\prime \prime}}\right), 22.522\left(\mathrm{C}_{9^{\prime \prime}}\right), 14.327\left(\mathrm{C}_{10^{\prime \prime}}\right)$.

6-Azauridine laurate: ${ }^{1} \mathrm{H} \mathrm{NMR}: \delta 12.25\left(1 \mathrm{H}\right.$, br s, $\left.\mathrm{H}_{3}\right)$, $7.52\left(1 \mathrm{H}, \mathrm{s}, \mathrm{H}_{5}\right), 5.90\left(1 \mathrm{H}, \mathrm{m}, \mathrm{H}_{1^{\prime}}\right), 5.38\left(1 \mathrm{H}, \mathrm{br} \mathrm{s}, \mathrm{OH}_{2^{\prime}}\right), 5.20$ $\left(1 \mathrm{H}\right.$, br s, $\left.\mathrm{OH}_{3^{\prime}}\right), 4.27-4.25\left(1 \mathrm{H}, \mathrm{m}, \mathrm{H}_{5^{\prime}-1}\right), 4.19\left(1 \mathrm{H}, \mathrm{m}, \mathrm{H}_{2^{\prime}}\right), 4.04$ $\left(1 \mathrm{H}, \mathrm{m}, \mathrm{H}_{3^{\prime}}\right), 3.97-3.91\left(2 \mathrm{H}, \mathrm{H}_{4^{\prime}}+\mathrm{H}_{5^{\prime}-2}\right), 2.25(2 \mathrm{H}, \mathrm{t}, J=8 \mathrm{~Hz}$, $\left.\mathrm{H}_{2^{\prime \prime}}\right), 1.47\left(2 \mathrm{H}, \mathrm{m}, \mathrm{H}_{3^{\prime \prime}}\right), 1.21\left(16 \mathrm{H}, \mathrm{m}, \mathrm{H}_{4^{\prime \prime}}+\mathrm{H}_{5^{\prime \prime}}+\mathrm{H}_{6^{\prime \prime}}+\mathrm{H}_{7^{\prime \prime}}+\mathrm{H}_{8^{\prime \prime}}\right.$ $\left.+\mathrm{H}_{9^{\prime \prime}}+\mathrm{H}_{10^{\prime \prime}}+\mathrm{H}_{11^{\prime \prime}}\right), 0.83\left(3 \mathrm{H}, \mathrm{t}, J=6.4 \mathrm{~Hz}, \mathrm{H}_{12^{\prime \prime}}\right) .{ }^{13} \mathrm{C} \mathrm{NMR}: \delta$ $173.174\left(\mathrm{C}_{1^{\prime \prime}}\right), 156.861\left(\mathrm{C}_{4}\right), 148.566\left(\mathrm{C}_{2}\right), 136.775\left(\mathrm{C}_{5}\right), 90.220$ $\left(\mathrm{C}_{1^{\prime}}\right), 81.314\left(\mathrm{C}_{4^{\prime}}\right), 72.964\left(\mathrm{C}_{2^{\prime}}\right), 70.996\left(\mathrm{C}_{3^{\prime}}\right), 64.447\left(\mathrm{C}_{5^{\prime}}\right), 33.798$ $\left(\mathrm{C}_{2^{\prime \prime}}\right), 31.746\left(\mathrm{C}_{10^{\prime \prime}}\right), 29.437-28.881\left(\mathrm{C}_{4^{\prime \prime}}+\mathrm{C}_{5^{\prime \prime}}+\mathrm{C}_{6^{\prime \prime}}+\mathrm{C}_{7^{\prime \prime}}+\mathrm{C}_{8^{\prime \prime}}+\right.$ $\left.\mathrm{C}_{9^{\prime \prime}}\right), 24.8142\left(\mathrm{C}_{3^{\prime \prime}}\right), 22.540\left(\mathrm{C}_{11^{\prime \prime}}\right), 14.340\left(\mathrm{C}_{12^{\prime \prime}}\right)$. 
6-Azauridine myristate: ${ }^{1} \mathrm{H}$ NMR: $\delta 12.18(1 \mathrm{H}$, br s, $\left.\mathrm{H}_{3}\right), 7.52\left(1 \mathrm{H}, \mathrm{s}, \mathrm{H}_{5}\right), 5.91\left(1 \mathrm{H}, \mathrm{d}, J=4 \mathrm{~Hz}, \mathrm{H}_{1^{\prime}}\right), 5.31-5.29$ $\left(2 \mathrm{H}, \mathrm{m}, \mathrm{OH}_{2^{\prime}}\right.$ and $\left.\mathrm{OH}_{3^{\prime}}\right), 4.27-4.25\left(1 \mathrm{H}, \mathrm{m}, \mathrm{H}_{5^{\prime}-1}\right), 4.21-4.19$ $\left(1 \mathrm{H}, \mathrm{m}, \mathrm{H}_{2^{\prime}}\right), 4.05-4.03\left(1 \mathrm{H}, \mathrm{m}, \mathrm{H}_{3^{\prime}}\right), 3.95-3.93\left(2 \mathrm{H}, \mathrm{m}, \mathrm{H}_{4^{\prime}}+\right.$ $\left.\mathrm{H}_{5^{\prime}-2}\right), 2.26\left(2 \mathrm{H}, \mathrm{t}, J=7.4 \mathrm{~Hz}, \mathrm{H}_{2^{\prime \prime}}\right), 1.48-1.46\left(2 \mathrm{H}, \mathrm{m}, \mathrm{H}_{3^{\prime \prime}}\right)$, $1.22\left(20 \mathrm{H}, \mathrm{m}, \mathrm{H}_{4^{\prime \prime}}+\mathrm{H}_{5^{\prime \prime}}+\mathrm{H}_{6^{\prime \prime}}+\mathrm{H}_{7^{\prime \prime}}+\mathrm{H}_{8^{\prime \prime}}+\mathrm{H}_{9^{\prime \prime}}+\mathrm{H}_{10^{\prime \prime}}+\mathrm{H}_{11^{\prime \prime}}+\right.$ $\left.\mathrm{H}_{12^{\prime \prime}}+\mathrm{H}_{13^{\prime \prime}}\right), 0.84\left(3 \mathrm{H}, \mathrm{t}, J=6.6 \mathrm{~Hz}, \mathrm{H}_{14^{\prime \prime}}\right) \cdot{ }^{13} \mathrm{C} \mathrm{NMR}: \delta 173.139$ $\left(\mathrm{C}_{1^{\prime}}\right), 156.929\left(\mathrm{C}_{4}\right), 148.632\left(\mathrm{C}_{2}\right), 136.794\left(\mathrm{C}_{5}\right), 90.261\left(\mathrm{C}_{1^{\prime}}\right)$, $81.316\left(\mathrm{C}_{4^{\prime}}\right), 72.981\left(\mathrm{C}_{2^{\prime}}\right), 71.029\left(\mathrm{C}_{3^{\prime}}\right), 64.483\left(\mathrm{C}_{5^{\prime}}\right), 33.806$ $\left(\mathrm{C}_{2^{\prime \prime}}\right), 31.759\left(\mathrm{C}_{12^{\prime \prime}}\right), 29.480-28.901\left(\mathrm{C}_{4^{\prime \prime}}+\mathrm{C}_{5^{\prime \prime}}+\mathrm{C}_{6^{\prime \prime}}+\mathrm{C}_{7^{\prime \prime}}+\mathrm{C}_{8^{\prime \prime}}+\right.$ $\left.\mathrm{C}_{9^{\prime \prime}}+\mathrm{C}_{10^{\prime \prime}}+\mathrm{C}_{11^{\prime \prime}}\right), 24.827\left(\mathrm{C}_{3^{\prime \prime}}\right), 22.546\left(\mathrm{C}_{13^{\prime \prime}}\right), 14.350\left(\mathrm{C}_{14^{\prime \prime}}\right)$.

6-Azauridine palmitate: ${ }^{1} \mathrm{H}$ NMR: $\delta 12.25(1 \mathrm{H}$, br s, $\left.\mathrm{H}_{3}\right), 7.55\left(1 \mathrm{H}, \mathrm{s}, \mathrm{H}_{5}\right), 5.90\left(1 \mathrm{H}, \mathrm{d}, J=4 \mathrm{~Hz}, \mathrm{H}_{1}\right), 5.39(1 \mathrm{H}, \mathrm{br}$ s, $\left.\mathrm{OH}_{2^{\prime}}\right), 5.21\left(1 \mathrm{H}\right.$, br s, $\left.\mathrm{OH}_{3^{\prime}}\right), 4.28-4.26\left(1 \mathrm{H}, \mathrm{m}, \mathrm{H}_{5^{\prime}-1}\right), 4.19$ $\left(1 \mathrm{H}, \mathrm{m}, \mathrm{H}_{2^{\prime}}\right), 4.05\left(1 \mathrm{H}, \mathrm{m}, \mathrm{H}_{3^{\prime}}\right), 3.98-3.92\left(2 \mathrm{H}, \mathrm{m}, \mathrm{H}_{4^{\prime}}+\mathrm{H}_{5^{\prime}-2}\right)$, $2.27\left(2 \mathrm{H}, \mathrm{t}, J=8 \mathrm{~Hz}, \mathrm{H}_{2^{\prime \prime}}\right), 1.50-1.47\left(2 \mathrm{H}, \mathrm{m}, \mathrm{H}_{3^{\prime \prime}}\right), 1.23(24 \mathrm{H}$, $\mathrm{m}, \mathrm{H}_{4^{\prime \prime}}+\mathrm{H}_{5^{\prime \prime}}+\mathrm{H}_{6^{\prime \prime}}+\mathrm{H}_{7^{\prime \prime}}+\mathrm{H}_{8^{\prime \prime}}+\mathrm{H}_{9^{\prime \prime}}+\mathrm{H}_{10^{\prime \prime}}+\mathrm{H}_{11^{\prime \prime}}+\mathrm{H}_{12^{\prime \prime}}+\mathrm{H}_{13^{\prime \prime}}+$ $\left.\mathrm{H}_{14^{\prime \prime}}+\mathrm{H}_{15^{\prime \prime}}\right), 0.85\left(3 \mathrm{H}, \mathrm{t}, J=8 \mathrm{~Hz}, \mathrm{H}_{16^{\prime \prime}}\right) .{ }^{13} \mathrm{C}$ NMR: $\delta 173.137$ $\left(\mathrm{C}_{1^{\prime \prime}}\right), 156.871\left(\mathrm{C}_{4}\right), 148.579\left(\mathrm{C}_{2}\right), 136.826\left(\mathrm{C}_{5}\right), 90.202\left(\mathrm{C}_{1^{\prime}}\right)$, $81.282\left(\mathrm{C}_{4^{\prime}}\right), 72.988\left(\mathrm{C}_{2^{\prime}}\right), 71.020\left(\mathrm{C}_{3^{\prime}}\right), 64.505\left(\mathrm{C}_{5^{\prime}}\right), 31.778\left(\mathrm{C}_{2^{\prime \prime}}\right)$, 29.534-28.921 $\left(\mathrm{C}_{4^{\prime \prime}}+\mathrm{C}_{5^{\prime \prime}}+\mathrm{C}_{6^{\prime \prime}}+\mathrm{C}_{7^{\prime \prime}}+\mathrm{C}_{8^{\prime \prime}}+\mathrm{C}_{9^{\prime \prime}}+\mathrm{C}_{10^{\prime \prime}}+\mathrm{C}_{11^{\prime \prime}}+\mathrm{C}_{12^{\prime \prime}}\right.$ $\left.+\mathrm{C}_{13^{\prime \prime}}+\mathrm{C}_{14^{\prime \prime}}\right), 24.838\left(\mathrm{C}_{3^{\prime \prime}}\right), 22.569\left(\mathrm{C}_{15^{\prime \prime}}\right), 14.376\left(\mathrm{C}_{16^{\prime \prime}}\right)$.

6-Azauridine chloroacetate: ${ }^{1} \mathrm{H}$ NMR: $\delta 12.19(1 \mathrm{H}$, br $\left.\mathrm{s}, \mathrm{H}_{3}\right), 7.52\left(1 \mathrm{H}, \mathrm{s}, \mathrm{H}_{5}\right), 5.89\left(1 \mathrm{H}, \mathrm{d}, J=4 \mathrm{~Hz}, \mathrm{H}_{1^{\prime}}\right), 5.36(1 \mathrm{H}$, br s, $\left.\mathrm{OH}_{2^{\prime}}\right), 5.20\left(1 \mathrm{H}\right.$, br s, $\left.\mathrm{OH}_{3^{\prime}}\right), 4.39-4.36\left(3 \mathrm{H}, \mathrm{m}, \mathrm{H}_{5^{\prime}-1}\right), 4.33$ $\left(1 \mathrm{H}, \mathrm{s}, \mathrm{H}_{2^{\prime}}\right), 4.17\left(1 \mathrm{H}, \mathrm{m}, \mathrm{H}_{2^{\prime}}\right), 4.08-4.01\left(1 \mathrm{H}, \mathrm{m}, \mathrm{H}_{3^{\prime}}\right), 3.98-$ $3.96\left(2 \mathrm{H}, \mathrm{m}, \mathrm{H}_{4^{\prime}}+\mathrm{H}_{5^{\prime}-2}\right) .{ }^{13} \mathrm{C} \mathrm{NMR}: \delta 167.640\left(\mathrm{C}_{\mathrm{1}^{\prime \prime}}\right), 156.895$ $\left(\mathrm{C}_{4}\right), 148.591\left(\mathrm{C}_{2}\right), 136.955\left(\mathrm{C}_{5}\right), 90.311\left(\mathrm{C}_{1^{\prime}}\right), 81.123\left(\mathrm{C}_{4^{\prime}}\right)$, $73.028\left(\mathrm{C}_{2^{\prime}}\right), 70.883\left(\mathrm{C}_{3^{\prime}}\right), 65.956\left(\mathrm{C}_{5^{\prime}}\right), 21.029\left(\mathrm{C}_{2^{\prime \prime}}\right)$.

6-Azauridine pivalate: ${ }^{1} \mathrm{H}$ NMR: $\delta 12.24\left(1 \mathrm{H}\right.$, br s, $\left.\mathrm{H}_{3}\right)$, $7.51\left(1 \mathrm{H}, \mathrm{s}, \mathrm{H}_{5}\right), 5.90\left(1 \mathrm{H}, \mathrm{m}, \mathrm{H}_{1^{\prime}}\right), 5.37\left(1 \mathrm{H}\right.$, br s, $\left.\mathrm{OH}_{2}\right), 5.19$ $\left(1 \mathrm{H}\right.$, br s, $\left.\mathrm{OH}_{3^{\prime}}\right), 4.22-4.20\left(1 \mathrm{H}, \mathrm{m}, \mathrm{H}_{5^{\prime}-1}\right), 4.20-4.18(1 \mathrm{H}, \mathrm{m}$, $\left.\mathrm{H}_{2^{\prime}}\right)$, 4.06-4.05 (1H, m, $\left.\mathrm{H}_{3^{\prime}}\right), 3.97-3.95\left(2 \mathrm{H}, \mathrm{m}, \mathrm{H}_{4^{\prime}}+\mathrm{H}_{5^{\prime}-2}\right), 1.11$ (9H, s, $\left.\mathrm{H}_{3^{\prime \prime}}\right) .{ }^{13} \mathrm{C}$ NMR: $\delta 177.926\left(\mathrm{C}_{1^{\prime \prime}}\right), 156.852\left(\mathrm{C}_{4}\right), 148.605$ $\left(\mathrm{C}_{2}\right), 136.800\left(\mathrm{C}_{5}\right), 90.063\left(\mathrm{C}_{1^{\prime}}\right), 81.283\left(\mathrm{C}_{4^{\prime}}\right), 72.856\left(\mathrm{C}_{2^{\prime}}\right)$, $70.764\left(\mathrm{C}_{3^{\prime}}\right), 64.395\left(\mathrm{C}_{5^{\prime}}\right), 38.687\left(\mathrm{C}_{2^{\prime \prime}}\right), 27.388\left(\mathrm{C}_{3^{\prime \prime}}\right), 27.358$ $\left(\mathrm{C}_{3^{\prime \prime}}\right), 27.267\left(\mathrm{C}_{3^{\prime \prime}}\right)$.

6-Azauridine crotonate: ${ }^{1} \mathrm{H}$ NMR: $\delta 12.22(1 \mathrm{H}$, br s, $\left.\mathrm{H}_{3}\right), 7.53$ (1H, s, $\left.\mathrm{H}_{5}\right), 6.91-6.84\left(1 \mathrm{H}, \mathrm{m}, \mathrm{H}_{3^{\prime \prime}}\right), 5.90-5.83(2 \mathrm{H}$, $\left.\mathrm{m}, \mathrm{H}_{1^{\prime}}+\mathrm{H}_{2^{\prime \prime}}\right), 5.37\left(1 \mathrm{H}\right.$, br s, $\left.\mathrm{OH}_{2^{\prime}}\right), 5.20\left(1 \mathrm{H}\right.$, br s, $\left.\mathrm{OH}_{3^{\prime}}\right), 4.33-$ $4.30\left(1 \mathrm{H}, \mathrm{m}, \mathrm{H}_{5^{\prime}-1}\right), 4.20\left(1 \mathrm{H}, \mathrm{m}, \mathrm{H}_{2^{\prime}}\right), 4.08-4.02\left(1 \mathrm{H}, \mathrm{m}, \mathrm{H}_{3^{\prime}}\right)$, 4.01-3.96 (2H, m, $\left.\mathrm{H}_{4^{\prime}}+\mathrm{H}_{5^{\prime}-2}\right), 1.84\left(3 \mathrm{H}, \mathrm{d}, J=6.8 \mathrm{~Hz}, \mathrm{H}_{4^{\prime \prime}}\right) .{ }^{13} \mathrm{C}$ NMR: $\delta 165.253\left(\mathrm{C}_{1^{\prime \prime}}\right), 156.369\left(\mathrm{C}_{4}\right), 148.083\left(\mathrm{C}_{2}\right), 145.545$ $\left(\mathrm{C}_{3^{\prime \prime}}\right), 136.322\left(\mathrm{C}_{5}\right), 121.912\left(\mathrm{C}_{2^{\prime \prime}}\right), 89.734\left(\mathrm{C}_{1^{\prime}}\right), 80.881\left(\mathrm{C}_{4^{\prime}}\right)$, $72.474\left(\mathrm{C}_{2^{\prime}}\right), 70.478\left(\mathrm{C}_{3^{\prime}}\right), 63.734\left(\mathrm{C}_{5^{\prime}}\right), 17.601\left(\mathrm{C}_{4^{\prime \prime}}\right)$.

6-Azauridine 10-undecenoate: ${ }^{1} \mathrm{H}$ NMR: $\delta 12.21(1 \mathrm{H}$, br s, $\left.\mathrm{H}_{3}\right), 7.50\left(1 \mathrm{H}, \mathrm{s}, \mathrm{H}_{5}\right), 5.88\left(1 \mathrm{H}, \mathrm{d}, J=4 \mathrm{~Hz}, \mathrm{H}_{\mathrm{1}^{\prime}}\right), 5.79$ $5.69\left(1 \mathrm{H}, \mathrm{m}, \mathrm{H}_{10^{\prime \prime}}\right), 5.34\left(1 \mathrm{H}\right.$, br s, $\left.\mathrm{OH}_{2^{\prime}}\right), 5.16\left(1 \mathrm{H}\right.$, br s, $\left.\mathrm{OH}_{3^{\prime}}\right)$, 4.96-4.87 (1H, m, $\left.\mathrm{H}_{11^{\prime \prime}}\right), 4.25-4.23\left(1 \mathrm{H}, \mathrm{m}, \mathrm{H}_{5^{\prime}-1}\right), 4.17-4.16$ $\left(1 \mathrm{H}, \mathrm{m}, \mathrm{H}_{2^{\prime}}\right), 4.03-4.01\left(1 \mathrm{H}, \mathrm{m}, \mathrm{H}_{3^{\prime}}\right), 3.93-3.90\left(2 \mathrm{H}, \mathrm{m}, \mathrm{H}_{4^{\prime}}+\right.$ $\left.\mathrm{H}_{5^{\prime}}-2\right), 2.23\left(2 \mathrm{H}, \mathrm{t}, J=8 \mathrm{~Hz}, \mathrm{H}_{2^{\prime \prime}}\right), 1.96-1.95\left(2 \mathrm{H}, \mathrm{m}, \mathrm{H}_{9^{\prime \prime}}\right)$, 1.47-1.44 (2H, m, $\left.\mathrm{H}_{3^{\prime \prime}}\right)$, 1.30-1.19 (16H, m, $\mathrm{H}_{4^{\prime \prime}}+\mathrm{H}_{5^{\prime \prime}}+\mathrm{H}_{6^{\prime \prime}}+$ $\left.\mathrm{H}_{7^{\prime \prime}}+\mathrm{H}_{8^{\prime \prime}}\right) .{ }^{13} \mathrm{C}$ NMR: $\delta 173.155\left(\mathrm{C}_{1^{\prime \prime}}\right), 156.861\left(\mathrm{C}_{4}\right), 148.578$ $\left(\mathrm{C}_{2}\right), 139.257\left(\mathrm{C}_{10^{\prime \prime}}\right), 136.807\left(\mathrm{C}_{5}\right), 115.010\left(\mathrm{C}_{11^{\prime \prime}}\right), 90.252\left(\mathrm{C}_{1^{\prime}}\right), 81.334$ $\left(\mathrm{C}_{4^{\prime}}\right), 72.90\left(\mathrm{C}_{2^{\prime}}\right), 71.029\left(\mathrm{C}_{3^{\prime}}\right), 64.469\left(\mathrm{C}_{5^{\prime}}\right), 33.814\left(\mathrm{C}_{9^{\prime \prime}}\right), 33.619$, $\left(\mathrm{C}_{2^{\prime \prime}}\right)$, 29.095-28.705 $\left(\mathrm{C}_{4^{\prime \prime}}+\mathrm{C}_{5^{\prime \prime}}+\mathrm{C}_{6^{\prime \prime}}+\mathrm{C}_{7^{\prime \prime}}+\mathrm{C}_{8^{\prime \prime}}\right), 24.823\left(\mathrm{C}_{3^{\prime \prime}}\right)$.

6-Azauridine benzoate: ${ }^{1} \mathrm{H}$ NMR: $\delta 12.22\left(1 \mathrm{H}\right.$, br s, $\left.\mathrm{H}_{3}\right)$, $7.95\left(2 \mathrm{H}, \mathrm{d}, J=8 \mathrm{~Hz}, \mathrm{H}_{3^{\prime \prime}}+\mathrm{H}_{7^{\prime \prime}}\right), 7.67-7.63\left(1 \mathrm{H}, \mathrm{m}, \mathrm{H}_{5^{\prime \prime}}\right), 7.54-$ $7.50\left(2 \mathrm{H}, \mathrm{m}, \mathrm{H}_{4^{\prime \prime}}+\mathrm{H}_{6^{\prime \prime}}\right), 7.38\left(1 \mathrm{H}, \mathrm{s}, \mathrm{H}_{5}\right), 5.95(1 \mathrm{H}, \mathrm{d}, J=4$
$\left.\mathrm{Hz}, \mathrm{H}_{1^{\prime}}\right), 5.42\left(1 \mathrm{H}\right.$, br s, $\left.\mathrm{OH}_{2}\right), 5.28\left(1 \mathrm{H}\right.$, br s, $\left.\mathrm{OH}_{3^{\prime}}\right), 4.54-4.50$ $\left(1 \mathrm{H}, \mathrm{m}, \mathrm{H}_{5^{\prime}-1}\right), 4.30-4.29\left(1 \mathrm{H}, \mathrm{m}, \mathrm{H}_{2^{\prime}}\right), 4.27-4.26\left(1 \mathrm{H}, \mathrm{m}, \mathrm{H}_{3^{\prime}}\right)$, 4.14-4.12 (2H, m, $\left.\mathrm{H}_{4^{\prime}}+\mathrm{H}_{5^{\prime}-2}\right) .{ }^{13} \mathrm{C}$ NMR: $\delta 165.488\left(\mathrm{C}_{1^{\prime \prime}}\right)$, $156.292\left(\mathrm{C}_{4}\right), 148.082\left(\mathrm{C}_{2}\right), 136.264\left(\mathrm{C}_{5}\right), 133.349\left(\mathrm{C}_{5^{\prime \prime}}\right)$, $129.460\left(\mathrm{C}_{3^{\prime \prime}}+\mathrm{C}_{7^{\prime \prime}}\right), 129.139\left(\mathrm{C}_{2^{\prime \prime}}\right), 128.684\left(\mathrm{C}_{4^{\prime \prime}}+\mathrm{C}_{6^{\prime \prime}}\right), 89.700$ $\left(\mathrm{C}_{1^{\prime}}\right), 80.846\left(\mathrm{C}_{4^{\prime}}\right), 72.555\left(\mathrm{C}_{2^{\prime}}\right), 70.350\left(\mathrm{C}_{3^{\prime}}\right), 64.207\left(\mathrm{C}_{5^{\prime}}\right)$.

\section{RESULTS AND DISCUSSION}

Effect of reaction medium: The performance of the enzyme is usually greatly affected by the nature of the medium ${ }^{16}$. As reported by our previous work, although acetone and THF have proved to be suitable organic solvents for the synthesis of esters derivatives of nucleosides, there exists some drawbacks (such as the strong polarity of the acetone or the poor results in THF) in the procedure of the enzymatic acylation ${ }^{13,17}$. As an alternative, co-solvent mixtures were tried. Considering that a majority of the enzymes were more stable in hydrophobic solvents, the acylation of 6-azauridine with vinyl 10-undecenoate was carried out in eight various binary mixtures of THFor acetone-miscible organic solvents (Table-1). As expected, the initial reaction rate and substrate conversion changed dramatically when the hydrophobic solvents were added into the pure solvent. Among the binary organic solvents tested, the addition of $25 \%(\mathrm{v} / \mathrm{v})$ isooctane to acetone resulted in a clear improvement in the initial reaction rate and maximum substrate conversion. The possible reason might be attributable to the reduced inactivation of the $C$. antarctica lipase B resulting from the lowering of the acetone polarity caused by addition of the hydrophobic organic solvent ${ }^{18}$.

TABLE-1

EFFECT OF ORGANIC SOLVENTS ON $C$. antarctica

LIPASE B -CATALYZED REGIOSELECTIVE ACYLATION OF AZURD WITH VINYL 10-UNDECENOATE ${ }^{a}$

\begin{tabular}{|c|c|c|c|c|}
\hline Solvents & $\begin{array}{c}\mathrm{V}_{0} \\
(\mathrm{mM} / \mathrm{h})\end{array}$ & $\begin{array}{l}\text { Time } \\
\text { (h) }\end{array}$ & $\begin{array}{l}\mathrm{C}^{\mathrm{b}} \\
(\%)\end{array}$ & $\begin{array}{l}\text { 5'-Regio- } \\
\text { selectivity } \\
(\%)\end{array}$ \\
\hline Acetone & 9.8 & 9.5 & 56.3 & 99 \\
\hline Acetone/isooctane $(75: 25)$ & 13.3 & 7.0 & 86.7 & 99 \\
\hline Acetone/isopropyl ether $(75: 25)$ & 11.8 & 8.0 & 86.0 & 99 \\
\hline Acetone/cyclohexane $(75: 25)$ & 7.4 & 11.5 & 84.2 & 99 \\
\hline Acetone/hexane $(75: 25)$ & 5.5 & 12.5 & 78.8 & 99 \\
\hline THF & 6.0 & 9.5 & 24.4 & 99 \\
\hline THF/isooctane 75:25) & 6.4 & 10.0 & 81.4 & 99 \\
\hline THF/isopropyl ether (75:25) & 8.0 & 10.5 & 84.0 & 99 \\
\hline THF/cyclohexane (75:25) & 5.8 & 12.0 & 74.1 & 99 \\
\hline THF/hexane (75:25) & 4.9 & 12.0 & 63.5 & 99 \\
\hline Acetone/isooctane (90:10) & 13.9 & 7.5 & 84.6 & 99 \\
\hline Acetone/isooctane (85:15) & 15.3 & 6.0 & 90.5 & 99 \\
\hline Acetone/isooctane $(80: 20)$ & 14.5 & 6.5 & 90.0 & 99 \\
\hline Acetone/isooctane (70:30) & 10.1 & 8.5 & 80.4 & 99 \\
\hline Acetone/isooctane $(65: 35)$ & 8.8 & 9.5 & 76.2 & 99 \\
\hline
\end{tabular}

${ }^{a}$ Reactions were carried out at $40{ }^{\circ} \mathrm{C}, 200 \mathrm{rpm}$ by adding $0.04 \mathrm{mmol} 6-$ azauridine, $0.04 \mathrm{mmol}$ vinyl 10-undecenoate, $29 \mathrm{U} \mathrm{C}$. antarctica lipase B into $2 \mathrm{~mL}$ anhydrous solvent or binary solvent mixtures. ${ }^{b}$ Maximum conversion of substrate

Moreover, the reaction rate and substrate conversion boosted markedly with decreasing the content of the isooctane $(10 \%)$. Nevertheless, a further rise in isooctane content beyond $15 \%(\mathrm{v} / \mathrm{v})$ led to a sharp drop in the reaction rate, suggesting that the lower solubility of 6-azauridine in binary mixtures 
TABLE-2

EFFECT OF $\mathrm{a}_{\mathrm{w}}$ ON $C$. antarctica LIPASE B-CATALYZED ACYLATION OF AZURD WITH VINYL 10-UNDECENOATE IN BINARY SOLVENT MIXTURES ${ }^{\mathrm{a}}$

\begin{tabular}{|c|c|c|c|c|}
\hline$a_{w}$ & $\mathrm{~V}_{0}(\mathrm{mM} / \mathrm{h})$ & Time (h) & $\mathrm{C}(\%)$ & 5'-Regio-selectivity (\%) \\
\hline$\sim 0^{\mathrm{b}}$ & 15.3 & 6.0 & 90.5 & 99 \\
\hline 0.07 & 3.7 & 5.0 & 35.6 & 99 \\
\hline 0.11 & 2.6 & 4.0 & 19.0 & 99 \\
\hline 0.23 & 0.3 & 3.0 & 10.7 & 99 \\
\hline 0.33 & 0.1 & 2.0 & 1.9 & 99 \\
\hline 0.55 & 0.1 & 2.0 & 1.1 & 99 \\
\hline 0.75 & n.d. & 2.0 & n.d. & n.d. \\
\hline \multicolumn{5}{|c|}{$\begin{array}{l}{ }^{2} \text { Reactions were carried out at } 40{ }^{\circ} \mathrm{C}, 200 \mathrm{rpm} \text { and various } a_{w} \text { values by } \\
\text { adding } 0.04 \mathrm{mmol} \text { AzUrd, } 0.04 \mathrm{mmol} \text { vinyl } 10 \text {-undecenoate, } 29 \mathrm{U} C \\
\text { antarctica lipase B into } 2 \mathrm{~mL} \text { anhydrous acetone/isooctane }(85: 15 \\
\mathrm{v} / \mathrm{v}) .{ }^{b} \text { Enzyme used as received }\end{array}$} \\
\hline
\end{tabular}

(data not shown) was responsible for this. It is worth-noting that the isooctane content showed little effect on the 5'-regioselectivity of the reaction.

Effect of initial water activity: It is well-documented that each enzyme has the specific water activity requirement, which allows the enzyme to ensure the conformational mobility required for biotransformation ${ }^{19}$. Furthermore, it is important to pay attention to water control since the presence of water may also facilitate the competitive hydrolysis of both the product and vinyl 10-undecenoate.

As shown in Table-2, a rather low initial reaction rate and 6-azauridine conversion were observed when the acylation was performed in higher aw solvent systems, indicating that the C. antarctica lipase B-mediated acylation of 6-azauridine displayed a clear $\mathrm{a}_{\mathrm{w}}$ dependence in the binary mixture of organic solvents. The best result was obtained in anhydrous acetone/isooctane $(85: 15, \mathrm{v} / \mathrm{v})$, suggesting that the water in the commercial enzyme preparation was enough to retain a high enzymatic activity. A more high $\mathrm{a}_{\mathrm{w}}$ value would result in the acceleration of the competitive hydrolysis mentioned above, thus leading to the poor results. Fortunately, the C. antarctica lipase B lipase displayed an absolute 5'-regioselectivity within the scope investigaed.

Effect of the ratio of vinyl 10-undecenoate to 6-azauridine: It was reported that the enzymatic acylation of substrate with vinyl ester was usually accompanied by the hydrolysis of the acyl donor ${ }^{20}$. The analysis of the data (Fig. 1) obtained established that the excessive amount of vinyl 10-undecenoate was necessary for this acylation. Striking enhancement in both the initial reaction rate and maximal conversion was observed with the increase of the ratio up to 3 , the optimal ratio of vinyl 10undecenoate to 6-azauridine. A further increase in the ratio showed no appreciable improvement in either parameter, which was in good agreement with our previous reports ${ }^{17}$.

Effect of reaction temperature: Temperature has great effect on the activity, selectivity and stability of a lipase and the reaction equilibrium as well. Fig. 2 depicted the prominent impact of reaction temperature on the acylation. Within the examined temperature range between 35 and $55{ }^{\circ} \mathrm{C}$, the reaction accelerated distinctly with the increase of reaction temperature, while the 5'-regioselectivity showed little variation. Further increase in temperature resulted in a notable drop in the reaction rate, suggesting the partial inactivation of the lipase at a higher temperature. In view of the initial reaction

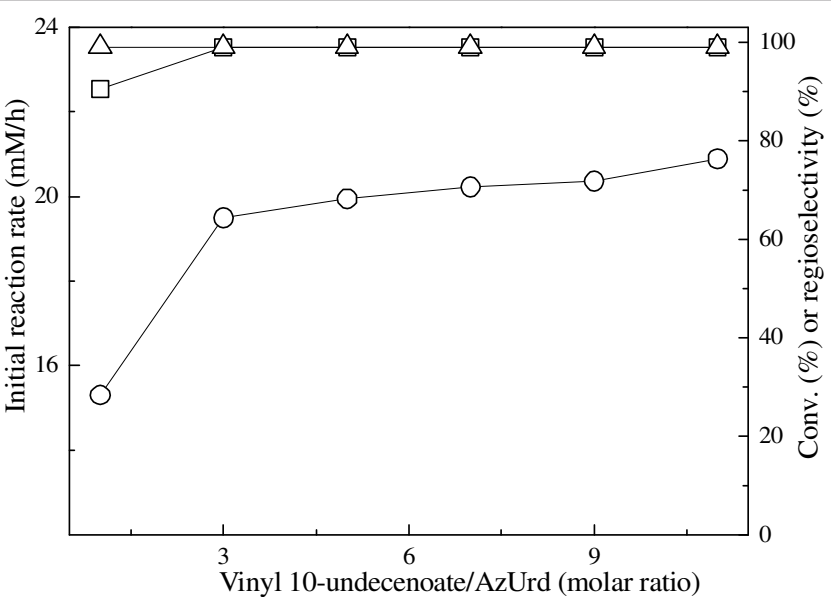

$\multimap-$ : Initial reaction rate; $-\square-$ : Maximum conv.; $\triangle$ : Regioselectivity

Fig. 1. Effect of the molar ratio of vinyl 10-undecenoate to 6-azauridine on $C$. antarctica lipase B-catalyzed acylation of 6-azauridine in binary solvent mixtures. The reactions were carried out at $40{ }^{\circ} \mathrm{C}$, $200 \mathrm{rpm}$ by adding $0.04 \mathrm{mmol} 6$-azauridine, various mole vinyl 10-undecenoate and $29 \mathrm{U} \mathrm{C}$. antarctica lipase B into $2 \mathrm{~mL}$ anhydrous acetone/isooctane (85:15, v/v)

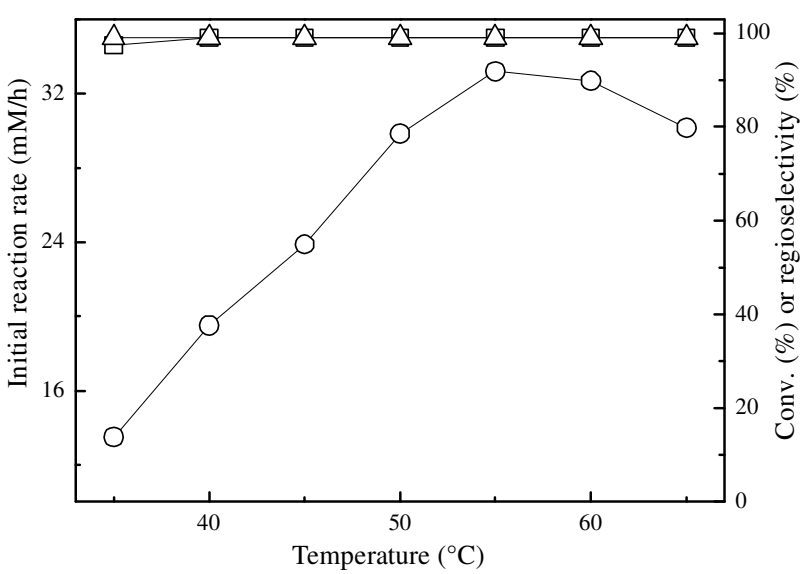

- - Initial reaction rate; $\square-$ : Maximum conv.; $\triangle$ : Regioselectivity

Fig. 2. Effect of the temperature on $C$. antarctica lipase B-catalyzed acylation of 6-azauridine in binary solvent mixtures. The reactions were carried out at $200 \mathrm{rpm}$, various temperatures from 35 to $65^{\circ} \mathrm{C}$ by adding $0.04 \mathrm{mmol} 6$-azauridine, $0.12 \mathrm{mmol}$ vinyl 10-undecenoate and $29 \mathrm{U} C$. antarctica lipase $\mathrm{B}$ into $2 \mathrm{~mL}$ anhydrous acetone/ isooctane $(85: 15, \mathrm{v} / \mathrm{v})$

rate, conversion and 5 '-regioselectivity, $55^{\circ} \mathrm{C}$ was selected as the optimum reaction temperature for this acylation.

Thermal and operational stability of the enzyme: From the points of view of the theoretical and practical implications, not only the initial rate and 5'-regioselectivity, but also the thermal and operational stabilities of $C$. antarctica lipase B were also significant criterions for efficient enzymatic acylation of 6-azauridine. Fig. 3 illustrated the residual activities of the C. antarctica lipase B after incubation in pure solvent and acetone/isooctane $(85 / 15, \mathrm{v} / \mathrm{v})$ for $5.5 \mathrm{~h}$ at various temperatures from $35-65^{\circ} \mathrm{C}$. Surprisingly, the much higher enzymatic activity of $60 \%$ in $15 \%(\mathrm{v} / \mathrm{v})$ isooctane-acetone at such a high temperature $\left(65^{\circ} \mathrm{C}\right)$ was observed than that in pure acetone $(38 \%)$. The addition of hydrophobic solvent to the polar reaction medium might be beneficial to maintain the spatial structure of the enzyme protein at higher temperatures. Additionally, $C$. antarctica lipase B presented much better 


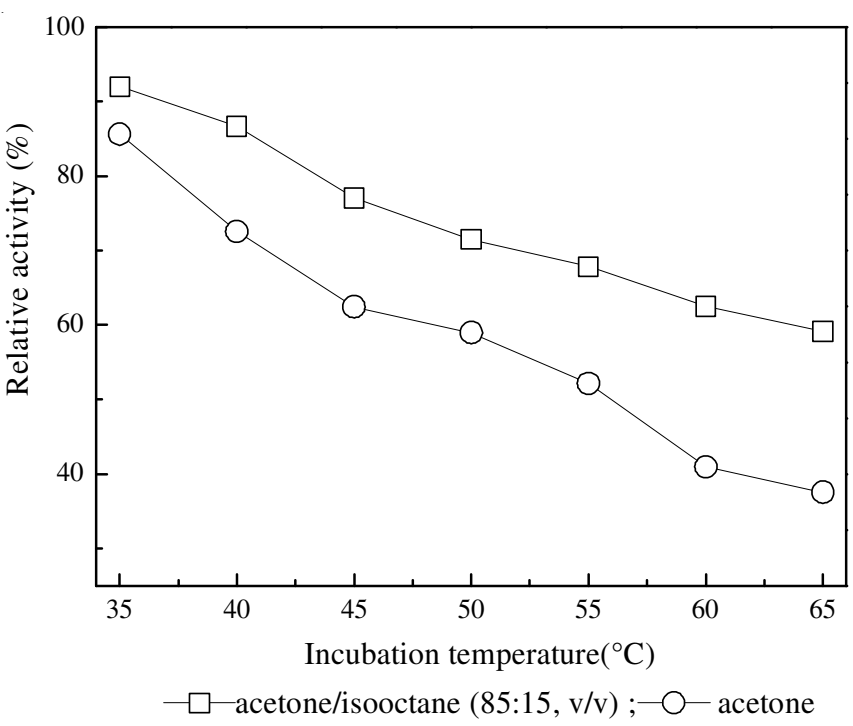

Fig. 3. Thermal stability of $C$. antarctica lipase B in different medium. The reactions were carried out at $55^{\circ} \mathrm{C}, 200 \mathrm{rpm}$ by adding 0.04 mmol 6-azauridine, $0.12 \mathrm{mmol}$ vinyl 10-undecenoate and $29 \mathrm{U} C$. antarctica lipase B into $2 \mathrm{~mL}$ anhydrous acetone/isooctane $(85 / 15$, $\mathrm{v} / \mathrm{v})$

operational stabilities in binary mixtures (acetone/isooctane) than in pure pyridine (Fig. 4). After being used repeatedly for 8 batches ( $5.5 \mathrm{~h}$ per batch) under the optimized reaction conditions, the enzyme still remained about $64 \%$ of their original activity in the binary solvent system, but only $39 \%$ residue activity was found in pure acetone. The coating and protection of the essential water of the enzyme protein by the isooctane molecules could partly account for this.

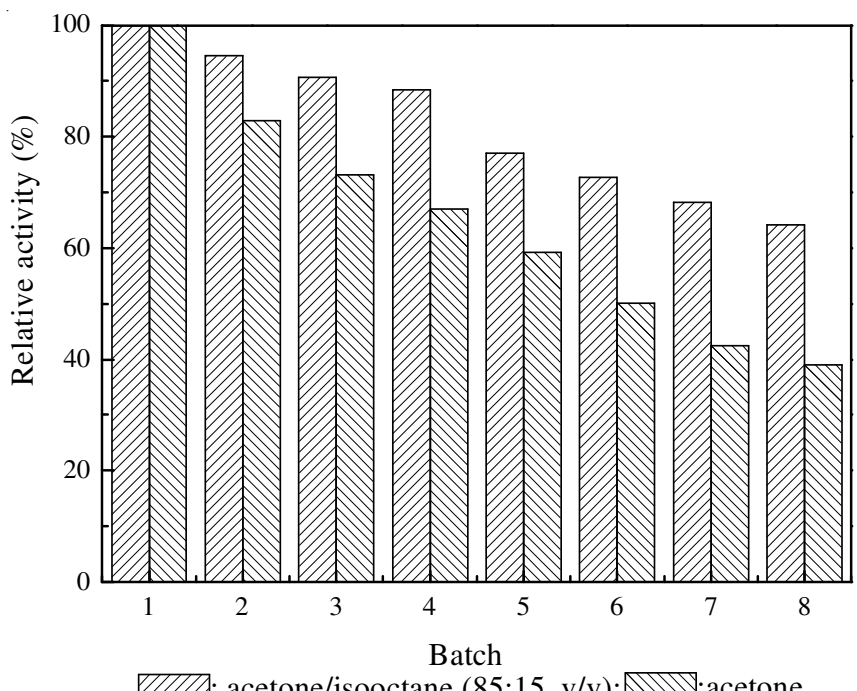

Fig. 4. Operational stability of $C$. antarctica lipase B in different medium. The reactions were carried out at $55^{\circ} \mathrm{C}, 200 \mathrm{rpm}$ by adding 0.04 mmol 6-azauridine, $0.12 \mathrm{mmol}$ vinyl 10-undecenoate and $29 \mathrm{U} C$. antarctica lipase B into $2 \mathrm{~mL}$ anhydrous acetone/isooctane $(85 / 15$, $\mathrm{v} / \mathrm{v})$

Effect of various acyl donors: In order to investigate the universality of the binary mixture of anhydrous acetone/ isooctane $(85: 15, \mathrm{v} / \mathrm{v})$ for synthesis of potential 6-azauridine prodrugs, various structural vinyl esters were used as the acylating agents for enzymatic acylation.
As can be seen in Table-3, the $C$. antarctica lipase B was highly effective in catalyzing the acylation of a series of saturate straight-chain vinyl esters (C2-C14) with 6-azauridine in the isooctane-based binary solvent system (Entries 1-8). Interestingly, the enzyme showed the highest catalytic activity toward vinyl butyrate $(\mathrm{C} 4)$ among the acyl donors tested (8 straight-chain fatty acid vinyl esters $(\mathrm{C} 2-\mathrm{C} 16)$ and 5 other carboxylic acid vinyl esters bearing substituents or functional groups) and was a good catalyst for the synthesis of shortchain fatty acid esters of 6-azauridine. However, the initial reaction rate lowered with the elongation of chain length of vinyl esters from $\mathrm{C} 4$ to $\mathrm{C} 16$, probably due to the acyl group of more than $\mathrm{C} 4$ would produce sharp distortion with the enzyme active site and result in an increase of the activation energy ${ }^{21}$. Additionally, it was noted that the substituent ( $\mathrm{Cl}$ atom) attached on the vinyl ester exerted a significant impact on the acylation with the unexpected reaction rate. The electron-withdrawing effect of the halogen substituent might decrease the electron density of carbonyl group and be advantageous to form acylenzyme intermediate ${ }^{22}$.

\begin{tabular}{|c|c|c|c|c|c|}
\hline \multicolumn{6}{|c|}{$\begin{array}{c}\text { TABLE-3 } \\
\text { EFFECT OF VARIOUS ACYL DONORS ON } C \text {. antarctica LIPASE } \\
\text { B -CATALYZED REGIOSELECTIVE ACYLATION } \\
\text { OF AZURD IN BINARY MIXTURES }{ }^{\mathrm{a}}\end{array}$} \\
\hline Entry & Acyl donor & $\begin{array}{c}\mathrm{V}_{0} \\
(\mathrm{mM} / \mathrm{h})\end{array}$ & $\begin{array}{l}\text { Time } \\
\text { (h) }\end{array}$ & $\mathrm{C}(\%)$ & $\begin{array}{c}\text { 5'-Regio- } \\
\text { selectivity (\%) }\end{array}$ \\
\hline 1 & Vinyl acetate (C2) & 57.0 & 2.5 & $>99.0$ & 99 \\
\hline 2 & Vinyl butyrate (C4) & 68.7 & 2.0 & $>99.0$ & 99 \\
\hline 3 & Vinyl hexanoate (C6) & 55.1 & 2.5 & $>99.0$ & 99 \\
\hline 4 & Vinyl carprylate $(\mathrm{C} 8)$ & 41.8 & 3.0 & $>99.0$ & 99 \\
\hline 5 & Vinyl decanoate (C10) & 36.2 & 4.0 & $>99.0$ & 99 \\
\hline 6 & Vinyl laurate (C12) & 32.1 & 4.0 & $>99.0$ & 99 \\
\hline 7 & Vinyl myristate (C14) & 29.4 & 4.5 & $>99.0$ & 99 \\
\hline 8 & Vinyl palmitate (C16) & 26.8 & 4.5 & $>99.0$ & 99 \\
\hline 9 & Vinyl chloroacetate & 190.0 & 1.0 & $>99.0$ & 99 \\
\hline 10 & Vinyl pivalate & $<0.1$ & 28.0 & 4.1 & 99 \\
\hline 11 & Vinyl crotonate & 3.4 & 36.0 & 80.5 & 99 \\
\hline 12 & Vinyl 10-undecenoate & 33.2 & 4.0 & $>99.0$ & 99 \\
\hline 13 & Vinyl benzoate & $<0.1$ & 30.0 & 5.8 & 99 \\
\hline
\end{tabular}

${ }^{a}$ Reactions were carried out at $55^{\circ} \mathrm{C}, 200 \mathrm{rpm}$ by adding $0.04 \mathrm{mmol} 6-$ azauridine, $0.12 \mathrm{mmol}$ acyl donor, $29 \mathrm{U}$ C. antarctica lipase B into 2 $\mathrm{mL}$ anhydrous acetone/isooctane $(85: 15, \mathrm{v} / \mathrm{v})$

Moreover, the $C$. antarctica lipase B-catalyzed acylation rate decreased clearly with acyl donors bearing a branchedchain (Entry 10, Table-3) and an unsaturated bond (Entries 11 and 13, Table-3) conjugated with the carbonyl group. These phenomena were mainly attributed to not only the steric hindrance or limited rotational freedom derived from the vinyl esters carrying methyl, phenyl or C-C double bond at the $\alpha$ position, but also the structure feature of the enzyme ${ }^{23}$. Fortunately, the effect of $\mathrm{C}-\mathrm{C}$ double bond in vinyl 10-undecenoate is negligible, due to the fact that no conjugated effect occurred because of the long distance between the double bond and carbonyl group. To be satisfied, the 5'-regioselectivity maintained $99 \%$ with all the used acyl donors owing to the special structure of the enzyme's binding site.

\section{Conclusion}

In conclusion, the efficiency of the $C$. antarctica lipase B-catalyzed acylation of 6-azauridine with vinyl 10-undecenoate 
could be substantially enhanced in the $15 \%(\mathrm{v} / \mathrm{v})$ isooctanebased binary solvent system as compared to pure acetone. Various hydrophobic solvents exerted significant but different effects on the reaction and obviously improved the thermal and operational stabilities of $C$. antarctica lipase B. The established binary solvent system was also effective for the regioselective acylation of different acyl donors. Undoubtedly, these findings will enrich the fundamentals of non-aqueous enzymology and provide a greener enzymatic alternative as compared with the traditional chemical procedures.

\section{ACKNOWLEDGEMENTS}

This research was sponsored by Qing Lan Project of Jiangsu Province, Natural Science Foundation of Jiangsu Province (No. BK2012243), Foundation of Jiangsu Provincial Engineering Laboratory for Advanced Materials of Salt Chemical Industry (SF201310), Foundation of Jiangsu Provincial Engineering Laboratory for Biomass Conversion and Process Integration (JPELBCPL2013005) and Natural Science Research Project of Higher Education of Jiangsu Province (No. 10KJB530001).

\section{REFERENCES}

1. J.M. Crance, N. Scaramozzino, A. Jouan and D. Garin, Antiviral Res., 58, 73 (2003).

2. S.M. Kim, J.H. Park, K.N. Lee, S.K. Kim, Y.J. Ko, H.S. Lee and I.S Cho, Antiviral Res., 96, 213 (2012).

3. J. Plevová and I. Janku, Biochem. Pharmacol., 20, 2071 (1971).
4. M.A. Zinni, L.E. Iglesias and A.M. Iribarren, J. Mol. Catal. B, 47, 86 (2007).

5. M. Ferrero and V. Gotor, Chem. Rev., 100, 4319 (2000).

6. A.M. Gumel, M.S.M. Annuar, T. Heidelberg and Y. Chisti, Process Biochem., 46, 2079 (2011).

7. Z.Y. Wang and M.H. Zong, Chin. J. Catal., 32, 357 (2011).

8. S. Ozaki, T. Akiyama, T. Morita, M. Kumegawa, T. Nagase, N. Uehara and A. Hoshi, Chem. Pharm. Bull. (Tokyo), 38, 3164 (1990).

9. Z.Y. Wang, Y.H. Bi, X.Q. Li and M.H. Zong, Process Biochem., 48, 1208 (2013).

10. Z.Y. Wang, Y.H. Bi and M.H. Zong, Biotechnol. Lett., 34, 55 (2012).

11. Z.Y. Wang, Y.H. Bi and M.H. Zong, Appl. Biochem. Biotechnol., 165, 1161 (2011).

12. T. Zendo, F. Yoneyama and K. Sonomoto, Appl. Microbiol. Biotechnol., 88, 1 (2010).

13. H. Bi, Z.Y. Wang, L.H. Nie and X.Q. Li, Asian J. Chem., 25, 10273 (2013).

14. X.F. Li, M.H. Zong and G.L. Zhao, Bioorg. Med. Chem. Lett., 20, 4125 (2010).

15. P.J. Halling, Enzyme Microb. Technol., 16, 178 (1994).

16. A.M. Klibanov, Nature, 409, 241 (2001).

17. Z.Y. Wang and M.H. Zong, Biotechnol. Prog., 25, 784 (2009).

18. H. Zhao, Y. Zhang, F. Lu, X. Bie, Z. Lu and H. Ning, J. Mol. Catal. B, 69, 107 (2011).

19. F. Chamouleau, D. Coulon, M. Girardin and M. Ghoul, J. Mol. Catal. B, 11, 949 (2001).

20. H.K. Weber, H. Weber and R.J. Kazlauskas, Tetrahedron Asymm., 10, 2635 (1999).

21. J. Pleiss, M. Fischer and R.D. Schmid, Chem. Phys. Lipids, 93, 67 (1998).

22. A. Bertinotti, G. Carrea, G. Ottolina and S. Riva, Tetrahedron, 50, 13165 (1994).

23. T. Kobayashi, S. Adachi and R. Matsuno, Biotechnol. Lett., 25, 3 (2003). 